\title{
Probing Two-Dimensional vs Three-Dimensional Molecular Aggregation in Metal-Free Tetraphenylporphyrin Thin Films by Optical Anisotropy
}

\author{
G. Bussetti, ${ }^{* \dagger}$ M. Campione, ${ }^{\ddagger}$ L. Ferraro, ${ }^{\S}$ L. Raimondo, ${ }^{\S}$ B. Bonanni, ${ }^{\|}$C. Goletti, ${ }^{\|}$M. Palummo, ${ }^{\|,}$ \\ C. Hogan, ${ }^{\|, \perp, \mathscr{I l}}$ L. Duò, ${ }^{\dagger}$ M. Finazzi, ${ }^{\dagger}$ and A. Sassella ${ }^{\S}$ \\ ${ }^{\dagger}$ Department of Physics, Politecnico di Milano, p.za Leonardo da Vinci 32, I-20133 Milan, Italy \\ ${ }^{\ddagger}$ Department of Earth and Environmental Sciences, Università Milano-Bicocca, p.za della scienza 4, I-20126 Milan, Italy \\ ${ }^{\S}$ Department of Materials Science, Università Milano-Bicocca, via R. Cozzi 55, I-20125 Milan, Italy \\ "Department of Physics and CNISM, Università Tor Vergata, via Ricerca Scientifica 1, I-00133 Rome, Italy \\ ${ }^{\perp}$ CNR-ISM, via del Fosso del Cavaliere 100, I-00133 Rome, Italy
}

Received: February 13, 2014

Revised: June 10, 2014

Published: July 1, 2014

\section{INTRODUCTION}

Porphyrins and porphyrinoid compounds represent, nowadays, building blocks for the fascinating world of organic electronics. ${ }^{1}$ To date, porphyrins have been used in switches, ${ }^{2}$ memories, ${ }^{3,4}$ spectroscopic markers, ${ }^{5}$ solar cells, ${ }^{6}{ }^{2}$ sensors, ${ }^{7,8}$ tailored molecular catalysts, ${ }^{9}$ engineered molecular nanostructures, ${ }^{10}$ and molecular spintronic devices. ${ }^{11,12}$ In some cases, marketable devices such as the photogated ion conductor, ${ }^{13}$ the photogated transistor, ${ }^{1}$ and memory devices have been produced. ${ }^{14}$ In addition, porphyrins show an excellent stability as a function of temperature ${ }^{3}$ and a great flexibility in their chemical synthesis and manipulation. These properties allow one to grow solid porphyrin samples via different strategies (sublimation in vacuum, Langmuir-Blodgett deposition, electrochemical growth, chemical casting) and to organize molecules in many architectures from two-dimensional (2D) films ${ }^{15}$ to confined one-dimensional chains ${ }^{16}$ or zero-dimensional dots. ${ }^{17}$ These confined structures can be studied by different techniques, such as scanning probe microscopies (SPM), X-ray diffraction, and spectroscopy (optical transmission and absorbance, photoemission, Raman). Among them, optical spectroscopy in the UV-vis range offers some advantages, due to the fact that (i) optical probes are nondestructive, (ii) optical spectroscopy is very sensitive to changes in the electronic structure of the organic aggregates, ${ }^{18}$ (iii) the porphyrin characteristic transitions involving the extended $\pi$-electron system are in the visible range, (iv) porphyrins show strong optical absorption, ${ }^{18}$ and (v) optical probes can be used in situ during the organic sample growth. ${ }^{19}$

In this panorama, metal-free tetraphenylporphyrin $\left(\mathrm{H}_{2} \mathrm{TPP}\right)$ deposited on highly oriented pyrolytic graphite (HOPG) represents a paradigmatic system to study the behavior of molecule-molecule and molecule-substrate interactions. On the one hand, $\mathrm{H}_{2}$ TPP is the basic molecule to synthesize more complex porphyrinic compounds, it has a sharp square symmetry, and it is stable up to around $670 \mathrm{~K}$; thus it can be easily sublimated in a vacuum. On the other hand, the graphite substrate plays a crucial role in the growth and characterization of thin organic films. Graphite offers several advantages: (i) HOPG has atomically flat surfaces; (ii) a clean surface is easily obtained by manual exfoliation; (iii) the surface is stable in air; (iv) HOPG is a conductive substrate suitable for scanning tunneling microscopy measurements; (v) HOPG surface possesses $C_{3}$ symmetry and, consequently, is isotropic from an optical point of view; (vi) the interaction between molecules and graphite occurs via weak van der Waals forces. ${ }^{10,20}$ 
Conversely, traditional semiconductor surfaces undergo considerable reconstructions leaving unsaturated dangling bonds where porphyrins may interact simultaneously with different sites so that the total interaction can be strong enough to quench molecular mobility across the surface. ${ }^{21}$ Moreover, the growth of porphyrins on metal surfaces affects their conformation and their functionality ${ }^{22}$ and free-base porphyrins can undergo a spontaneous metalation. ${ }^{23}$

Figure 1 shows the structure of a single $\mathrm{H}_{2}$ TPP molecule: four external phenyl rings are covalently linked to a central

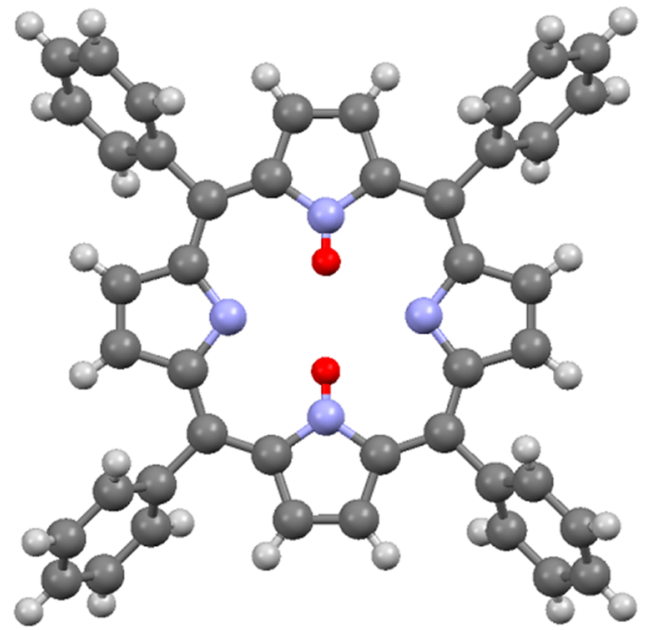

Figure 1. Structure of the metal-free tetraphenylporphyrin $\left(\mathrm{H}_{2} \mathrm{TPP}\right)$. The position of the two hydrogen atoms in the inner cavity of the molecule, red spheres, indicates that this is the structure of the tautomer frozen along the vertical direction. The molecule occupies a square surface with an about $12.2 \AA$ long side.

pyrrole cavity where two of the four nitrogen atoms (blue spheres in the figure) are bonded to a couple of hydrogen atoms (red spheres). It is well-known that the molecular symmetry allows these two $\mathrm{H}$ atoms to resonate between the two couples of adjacent opposing $\mathrm{N}$ atoms, creating a special molecular isomer called a tautomer. ${ }^{24}$ This proton transfer mechanism, called tautomerization, is inactive at temperatures below $240 \mathrm{~K}^{2}$ This quenching stabilizes one of the two tautomers, with the protons inside the inner cavity of the molecule along a preferential direction, resulting in a reduced symmetry and inducing porphyrins to acquire anisotropic electronic and optical properties, as evident in the theoretical optical response calculated at $T=0 \mathrm{~K}^{25}$

At room temperature (RT), tautomerization is active in the vapor phase and sometimes in the solid state, depending on the three-dimensional (3D) crystal structure. ${ }^{26}$ The bistability of the two hydrogen atoms between the two orthogonal alignments makes the individual $\mathrm{H}_{2}$ TPP molecule optically isotropic. Nevertheless, an optical anisotropy signal has been detected from porphyrin films deposited on HOPG at room temperature. ${ }^{27}$ In a thin film, the anisotropy could also arise from the molecular packing in the solid, triggered by the molecule-substrate and molecule-molecule interactions. ${ }^{28}$ Recently, however, it has been definitively demonstrated that a pure $2 \mathrm{D}$ layer of $\mathrm{H}_{2}$ TPP grown on graphite shows a square lattice molecular packing and that the anisotropic optical signal is associated with quenched tautomerization. ${ }^{29}$ In nominally thicker films, the $2 \mathrm{D}$ layer coexists with $3 \mathrm{D}$ crystallites, and it is interesting therefore to study the optical response of such films, in particular their anisotropy, with respect to that of the pure $2 \mathrm{D}$ phase that is fully dominated by the effects of tautomer alignment.

In this work, we address this subject by studying at first a reproducible way to prepare a purely $2 \mathrm{D}$ reference layer, starting from a thin film grown by organic molecular beam epitaxy (OMBE) on graphite. We then precisely characterize such a layer and compare its optical response with theoretical calculations. Finally, we analyze the morphology and the optical anisotropy spectra of $\mathrm{H}_{2}$ TPP thin films of different thicknesses, proposing a phenomenological model for the optical spectra and extracting the contributions of the $2 \mathrm{D}$ and $3 \mathrm{D}$ phases, with the latter being present in different amounts when the nominal film thickness is varied.

\section{EXPERIMENTAL DETAILS}

A high-quality $10 \times 10 \mathrm{~mm}^{2}$ HOPG slab (from Optigraph) has been used as substrate for deposition of porphyrin thin films, after exfoliation with an adhesive tape along the same direction before each experiment. $\mathrm{H}_{2} \mathrm{TPP}$ molecules (Sigma-Aldrich, purity $\geq 99 \%$ ) were deposited on HOPG by OMBE (base pressure $2 \times 10^{-7} \mathrm{mbar}$ ). For this purpose, a degassed quartz crucible has been held at $550 \mathrm{~K}$, while the HOPG has been kept at RT. The nominal thickness of the molecular film is determined by a quartz microbalance, mounted close to the graphite substrate during the sample growth. Atomic force microscopy (AFM) images have been collected in air by a Multimode Veeco instrument in noncontact operation mode, after the extraction of the sample from the growing chamber. A high-sensitivity (down to $10^{-6}$ ) homemade reflectance anisotropy spectroscopy (RAS) apparatus, ${ }^{30}$ with a light spot diameter of about $1 \mathrm{~mm}$, has been used to collect optical spectra in the $300-800 \mathrm{~nm}$ range with $2 \mathrm{~nm}$ spectral resolution. The integration time of each single experimental point is 100 ms. As the main porphyrin transition (Soret band) is in the visible range, we report RAS spectra in the reduced 400-500 $\mathrm{nm}$ range. In $\mathrm{RAS}^{31}{ }^{31}$ the linear polarization of light impinging onto the sample is modulated between two orthogonal directions $(\alpha$ and $\beta$ ). Such a modulation is obtained via the stress-induced birefringence of a photoelastic modulator. A phase-sensitive lock-in amplifier detects the optical anisotropy of the sample $\Delta R=R_{\alpha}-R_{\beta}$, where $R_{\alpha}$ and $R_{\beta}$ are the reflectances of the sample measured for light polarized along the mutually perpendicular $\alpha$ and $\beta$ directions, respectively. The result of the RAS measurements is expressed by the ratio $\Delta R / R$ between $\Delta R$ and the mean sample reflectance $R$. A sample azimuthal analysis is requested in order to find the maximum optical anisotropy. This procedure basically consists of acquiring RAS spectra as a function of azimuthal angle of the sample. The intensity of the optical signal commonly follows a $\cos 2 \theta$ law if the sample dielectric function is anisotropic, where $\theta$ is the azimuthal angle. This analysis is required if the sample does not possess well-defined crystal directions that are known a priori, as in our case. We find that the RAS signal is maximized (positive value) when the polarized electric fields are aligned parallel $(\alpha)$ and orthogonal $(\beta)$ to the graphite exfoliation direction, respectively. Indeed, graphite exfoliation breaks the substrate $C_{3}$ symmetry by the formation of monatomic steps and defects in a specific direction, so that it can induce some preferential arrangement of the overlayer. ${ }^{29}$ All the reported RAS data have been collected in this optical configuration. 

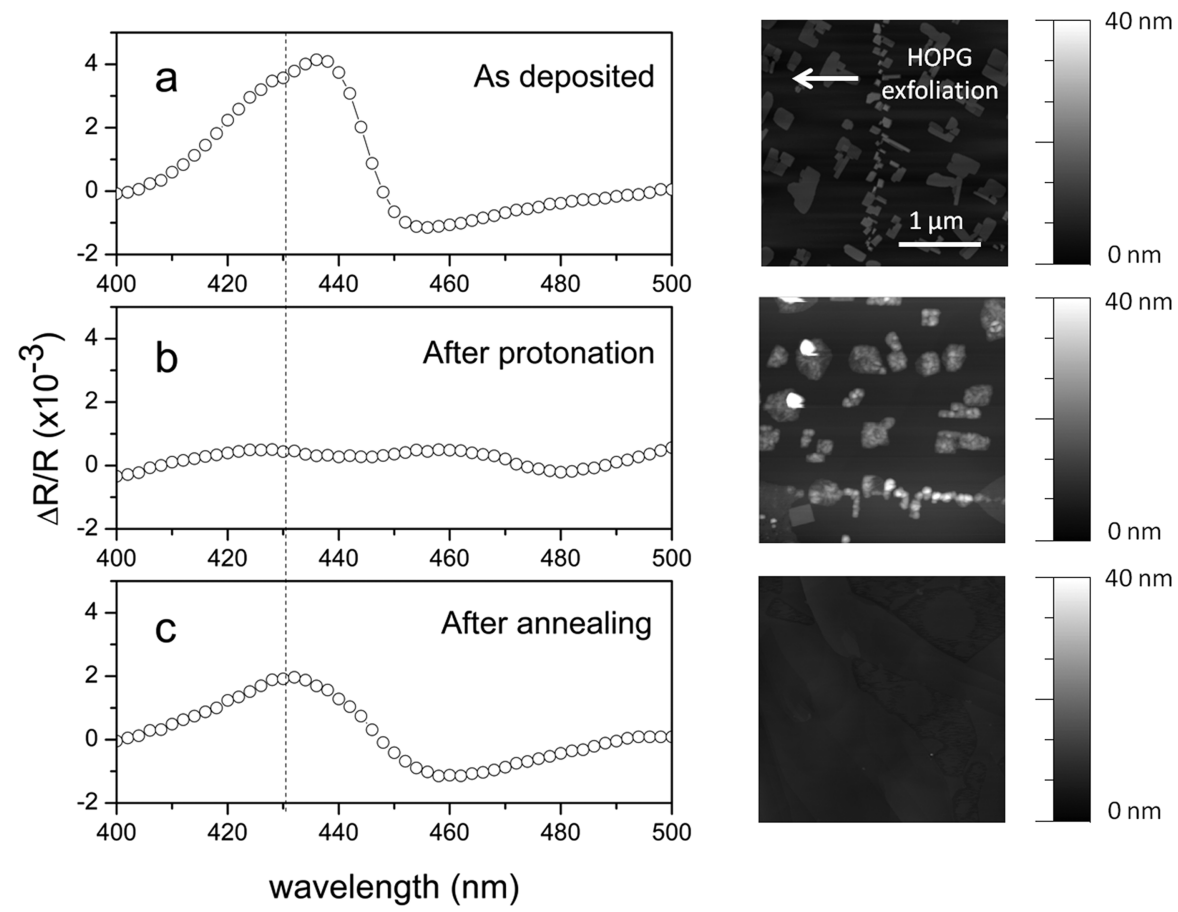

Figure 2. (a) Experimental RAS spectrum of a porphyrin thin film of $1 \mathrm{~nm}$ nominal thickness, with the corresponding AFM image collected over a 3 $\times 3 \mu \mathrm{m}^{2}$ wide region. (b) Experimental RAS spectrum of the same porphyrin thin film as in (a) after $\mathrm{HCl}$ exposure, with the corresponding AFM image collected over a $3 \times 3 \mu \mathrm{m}^{2}$ wide region. (c) Experimental RAS spectrum of the same porphyrin thin film as in (b) after several exposures to $\mathrm{HCl}$ vapors and a final annealing at $60^{\circ} \mathrm{C}$ for few seconds, with the corresponding AFM image collected over a $3 \times 3 \mu \mathrm{m}^{2}$ wide region.

$\mathrm{Ab}$ initio density functional theory (DFT) and many-body perturbation theory (MBPT) simulations of the electronic and optical properties were performed for the two-dimensional molecular layer, in a packing geometry consistent with ref 29 , where computational details can be found. In order to perform excited state MBPT calculations with the many-body code YAMBO, ${ }^{32}$ norm-conserving instead of soft pseudopotentials have been used. On top of DFT ground-state simulations, quasi-particle energies have been calculated within the perturbative GW method and then optical spectra simulations have been performed by means of the solution of the BetheSalpeter equation which describes the coupled electron-hole excitations. $^{25,33,34}$

\section{RESULTS AND DISCUSSION}

It has been already demonstrated that the $\mathrm{H}_{2}$ TPP film growth on HOPG follows a Stranski-Krastanov mode. ${ }^{29,35}$ Small 3D clusters, placed above a porphyrin 2D phase (wetting layer), have been always observed in the AFM images of all deposited films with different nominal thicknesses. As an example, in Figure 2a, we report both the RAS spectrum of a $\mathrm{H}_{2}$ TPP film with nominal deposited thickness of $1 \mathrm{~nm}$ and the corresponding AFM image. Here, many 3D structures are indeed visible, characterized by sharp edges, typical of crystallites. These structures can be grouped in two main categories: (i) some have a mean height of about $7 \mathrm{~nm}$ and a lateral size of about $400 \mathrm{~nm}$, while (ii) the smaller ones show the same height but a mean size of about $150 \mathrm{~nm}$ and are preferentially aligned along the graphite steps. Statistically, the $3 \mathrm{D}$ crystals cover almost $20 \%$ of the sample surface. Apparently, the concurrent presence on the surfaces of both the $2 \mathrm{D}$ and $3 \mathrm{D}$ porphyrin phases hinders the possibility to disentangle the characteristic optical properties of the wetting layer from those related to the porphyrin crystals. Consequently, the film measured anisotropy, which shows an asymmetric peak with a maximum close to $440 \mathrm{~nm}$, is in principle due both to the $2 \mathrm{D}$ layer and 3D structures.

Indeed, to analyze in depth the optical anisotropy response of the thin film, we are obliged to isolate the response of the two phases. One possibility is to prepare a purely $2 \mathrm{D}$ layer by following the two-step procedure pointed out and discussed in ref 29, and then characterize its optical response, which becomes a sort of RAS reference. In fact, a direct comparison between a RAS spectrum acquired on a porphyrin film, not previously treated by the two-step procedure, and the signal related to the undoubted purely $2 \mathrm{D}$ porphyrin layer allows evaluating if and to what extent the optical properties of the sample are dominated by the $2 \mathrm{D}$ phase and/or influenced by crystallites. We therefore repeatedly exposed the 1-nm-thick film in Figure 2a to $\mathrm{HCl}$ and then thermally treated the film at $60{ }^{\circ} \mathrm{C}$. After each step, we monitored the RAS response and the AFM surface morphology of the film, as shown in Figure 2b,c. After $\mathrm{HCl}$ exposure, which results in molecule protonation ${ }^{36}$ (incompatible with tautomerism), we observe partial dissolution of the 3D crystallites and an almost null RAS signal; after the thermal treatment, the optical anisotropy is recovered, albeit with a different, more symmetric line shape, and no 3D structures are now visible. Having thus identified the optimal preparation technique to get a purely $2 \mathrm{D}$ layer, we apply the same two-step method to the thinnest film we could grow, where very few and small 3D structures are present.

After growing a film as thin as $0.05 \mathrm{~nm}$ nominal thickness (to be interpreted here simply as a parameter indicating the total mass deposited onto the substrate) and treating it as described above, the results of optical and AFM characterization are reported in Figure 3a. The surface morphology only shows a 2D, molecularly flat layer. The corresponding RAS spectrum can therefore be considered as characteristic of such a phase. It 

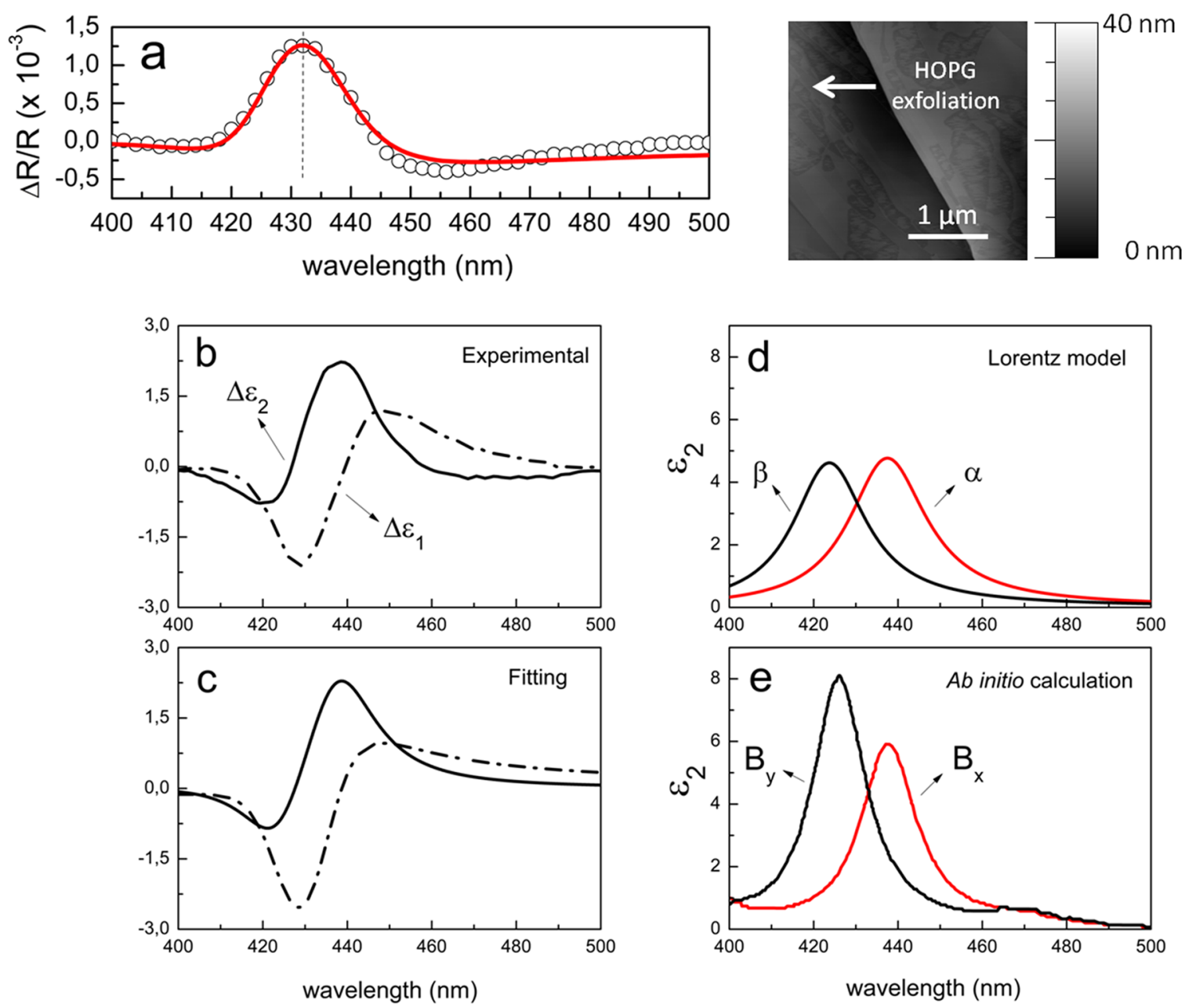

Figure 3. (a) Experimental RAS spectrum of a pure $2 \mathrm{D}$ porphyrin layer obtained from a thin film of $0.05 \mathrm{~nm}$ nominal thickness, after $\mathrm{HCl}$ exposure and thermal treatment at $60{ }^{\circ} \mathrm{C}$ for few seconds, with the corresponding AFM image collected over a $3 \times 3 \mu \mathrm{m}^{2}$ wide region. (b) Experimental $\Delta \varepsilon_{1}$ and $\Delta \varepsilon_{2}$ dielectric function anisotropies of the porphyrin 2D layer, as extracted from the experimental RAS spectrum in (a) following the three-layer model and using KK relations. (c) Best fit $\Delta \varepsilon_{1}$ and $\Delta \varepsilon_{2}$ dielectric function anisotropies of the porphyrin $2 \mathrm{D}$ layer, as extracted by fitting the experimental spectra in (b) using two Lorentz oscillators; the corresponding best fit of the RAS spectrum is reported in (a) as a red line. (d) $\varepsilon_{2}$ corresponding to the two best fit Lorentz oscillators used to obtain the $\Delta \varepsilon_{1}$ and $\Delta \varepsilon_{2}$ dielectric function anisotropies of the porphyrin $2 \mathrm{D}$ layer. (e) $\mathrm{Ab}$ initio calculated $\varepsilon_{2}$ for a $2 \mathrm{D}$ square domain of porphyrin molecules, characterized by frozen tautomers all aligned along the same direction; the labels are chosen accordingly to refs 25 and 29.

presents a single, highly symmetric peak centered at $432 \mathrm{~nm}$, with a small negative contribution at higher wavelengths. Given the homogeneous morphology of the treated 2D layer and knowing its structure as achieved by scanning tunneling microscopic analysis, ${ }^{29}$ it is possible to address a deeper interpretation and modeling of its optical anisotropy, as measured by RAS, in terms of the porphyrin layer dielectric function and Lorentz oscillators, respectively. To this end, the optical analysis requires some preliminary steps. In fact, when the RAS signal is acquired above the band gap of the substrate, as in this case, the optical anisotropy of the deposited layer has to be extracted from a spectrum, influenced by the optical properties of the buried substrate. Within the three-layer model, ${ }^{37}$ in which the substrate, the organic layer, and the environment are described as distinct phases characterized by specific dielectric functions, and in the limit of a small layer thickness, the RAS signal $\Delta R / R$ can be expressed in terms of the dielectric function of the anisotropic layer (the porphyrin 2D layer, in our case):

$$
\Delta R / R=(8 \pi d / \lambda)\left(A \Delta \varepsilon_{2}-B \Delta \varepsilon_{1}\right)
$$

Here, $\Delta \varepsilon_{2}=\left[\varepsilon_{2}\right]_{\alpha}-\left[\varepsilon_{2}\right]_{\beta}$ and $\Delta \varepsilon_{1}=\left[\varepsilon_{1}\right]_{\alpha}-\left[\varepsilon_{1}\right]_{\beta}$ are the anisotropy in the imaginary and real parts of $\varepsilon_{\text {layer }}$ along the $\alpha$ and $\beta$ directions, respectively. In eq $1, d$ is the film thickness and $\lambda$ is the wavelength of the impinging light (the mentioned low thickness approximation requires $d \ll \lambda$ ). $A$ and $B$ are characteristic functions of the substrate (HOPG, in our case) and they are expressed in terms of its dielectric function $\varepsilon_{\text {sub }}=$ $\varepsilon_{1 \text { sub }}+\mathrm{i} \varepsilon_{2 \text { sub }}$

$$
\begin{gathered}
A=\left(\varepsilon_{1 \mathrm{sub}}-1\right) /\left[\left(1-\varepsilon_{1 \mathrm{sub}}\right)^{2}+\varepsilon_{2 \mathrm{sub}}^{2}\right], \\
B=\varepsilon_{2 \mathrm{sub}} /\left[\left(1-\varepsilon_{1 \mathrm{sub}}\right)^{2}+\varepsilon_{2 \mathrm{sub}}^{2}\right]
\end{gathered}
$$

The $A$ and $B$ functions can be easily calculated from data available in the literature. ${ }^{38}$ From eq 1 , the RAS signal is seen to be the sum of two weighted contributions, namely the anisotropy of the dispersive $\left(\Delta \varepsilon_{1}\right)$ and the absorptive $\left(\Delta \varepsilon_{2}\right)$ porphyrin layer dielectric functions. Since $\Delta \varepsilon_{1}$ is connected to $\Delta \varepsilon_{2}$ by the Kramers-Kronig (KK) relations, ${ }^{39}$ eq 1 can be used to obtain $\Delta \varepsilon_{1}\left(\Delta \varepsilon_{2}\right)$ from the experimental data and by applying $\mathrm{KK}$ analysis, performed on an extended experimental spectral range. In Figure $3 \mathrm{~b}$, the so-obtained spectra of $\Delta \varepsilon_{1}$ and 
Table 1. Best Fit Parameters Obtained for RAS Spectra of Different Films: Position, Width, and Intensity of Lorentz Oscillators $\mathrm{LO}_{1}$ and $\mathrm{LO}_{2}$ Describing the Purely $2 \mathrm{D}$ film and the Same Parameters of the Voigt Curves $\mathrm{V}_{2 \mathrm{D}}$ and $\mathrm{V}_{3 \mathrm{D}}$ Accounting for the 2D and 3D Phases, Respectively, for the Thicker Films ${ }^{a}$

\begin{tabular}{|c|c|c|c|c|c|}
\hline nominal film thickness (nm) & dimensionality & & position $^{b}(\mathrm{~nm})$ & $\mathrm{fwhm}^{b, c}(\mathrm{~nm})$ & intensity \\
\hline 0.05 & purely $2 \mathrm{D}$ & $\mathrm{LO}_{1}$ & 424 & 19 & $4.6 \pm 0.1$ \\
\hline 0.05 & purely $2 \mathrm{D}$ & $\mathrm{LO}_{2}$ & 438 & 21 & $4.8 \pm 0.1$ \\
\hline 0.2 & mostly $2 \mathrm{D}$ & $\mathrm{V}_{2 \mathrm{D}}$ & 430 & 17 & $(5.2 \pm 0.4) \times 10^{-3}$ \\
\hline 1 & $2 \mathrm{D}$ phase & $\mathrm{V}_{2 \mathrm{D}}$ & 430 & 16 & $(3.8 \pm 0.4) \times 10^{-3}$ \\
\hline 1 & 3D phase & $\mathrm{V}_{3 \mathrm{D}}$ & 439 & 8 & $(2.6 \pm 0.4) \times 10^{-3}$ \\
\hline 5 & prevailing 3D & $\mathrm{V}_{3 \mathrm{D}}$ & 444 & 21 & $(41 \pm 1) \times 10^{-3}$ \\
\hline
\end{tabular}

${ }^{a}$ The corresponding best fit curves are reported as red curves in Figures $3 \mathrm{a}$ and 4 for the different films. ${ }^{b} \Delta \lambda=2 \mathrm{~nm}$. ${ }^{c}$ Full width at half-maximum.

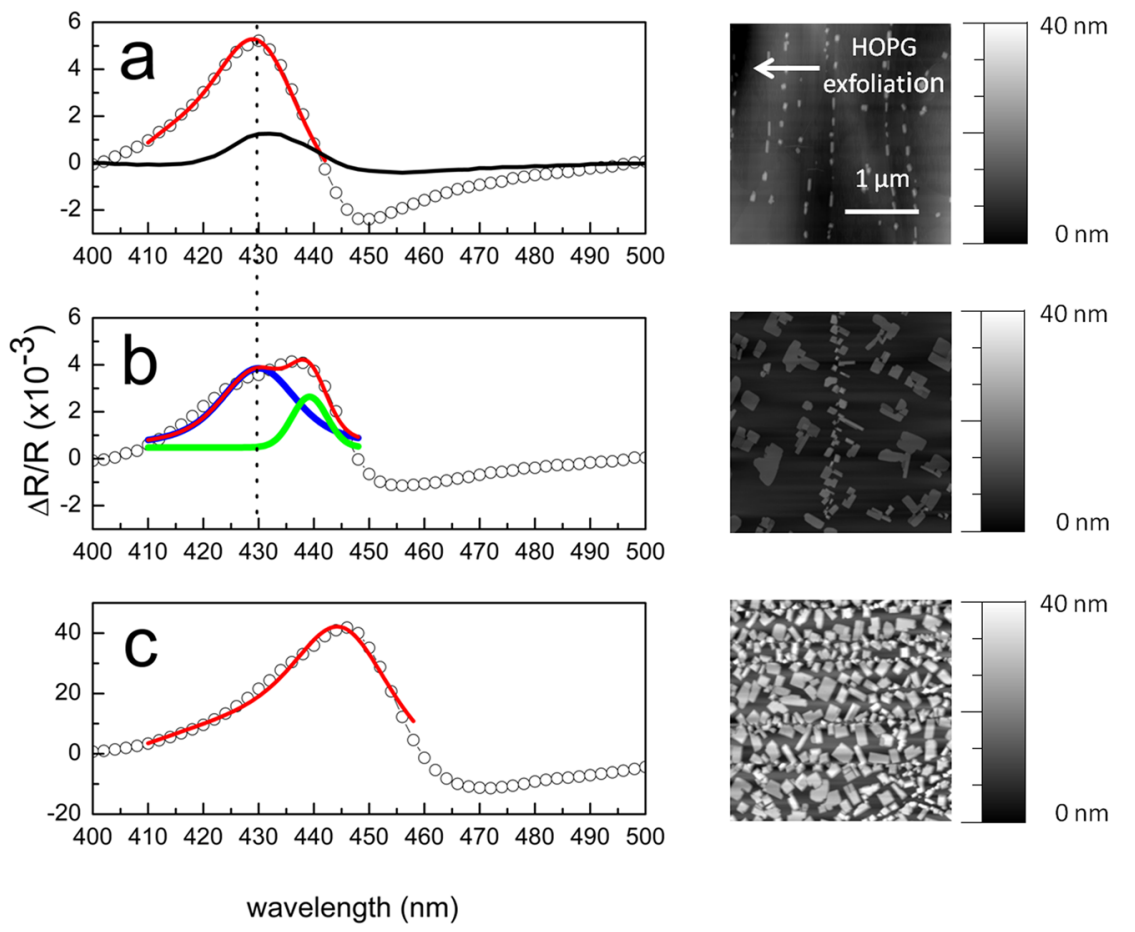

Figure 4. Experimental RAS spectra of porphyrin thin films of (a) 0.2 , (b) 1 , and (c) $5 \mathrm{~nm}$ nominal thickness, with the corresponding AFM images collected over $3 \times 3 \mu \mathrm{m}^{2}$ wide regions. In panel a, the solid black line refers to the RAS spectrum of a pure 2D phase for a direct comparison of the line shapes. The best fit curves obtained for the RAS spectra are reported as red lines; the one in (b) is the sum of two Voigt curves, the one centered at $430 \mathrm{~nm}$ (blue line) and the other one at $439 \mathrm{~nm}$ (green line).

$\Delta \varepsilon_{2}$ are reported in the spectral range of interest, from 400 to $500 \mathrm{~nm}$.

Considering that a single porphyrin molecule is characterized by two main energetically separated optical transitions, which are preferentially excited by perpendicularly oriented linearly polarized light, ${ }^{25,29}$ we have fitted the RAS spectrum by using two Lorentz oscillators (LOs) in order to reproduce the experimental $\Delta \varepsilon_{1}$ and $\Delta \varepsilon_{2}$ values. The best fit result is reported in terms of $\Delta \varepsilon_{1}$ and $\Delta \varepsilon_{2}$ in Figure 3c, showing a good agreement with the experimental data. Furthermore, to allow a detailed comparison with ab initio theoretical results, we report in Figure 3d the $\varepsilon_{2}$ value for each of the two Lorentz oscillators. These are found to well reproduce the components of the imaginary part of the dielectric tensor (shown in Figure 3e) for light polarized parallel and perpendicularly to the $\mathrm{H}-\mathrm{H}$ direction (which are equivalent to the experimental $\alpha$ and $\beta$ directions) calculated by the $\mathrm{ab}$ initio approaches described above, for a $2 \mathrm{D}$ layer of porphyrin molecules in a packing geometry consistent with that deduced in ref 29 . We note that the $\mathrm{ab}$ initio simulations fully include intermolecular interactions but only partially take into account the interaction between the molecular layer and the HOPG substrate. Structural (buckling) effects are included, but dielectric screening by the substrate is neglected. For this reason, the energy scale of the theoretical spectra has to be considered as relative. We have applied a rigid shift $(-0.3 \mathrm{eV})$ to align the calculate spectral features with the experimental ones. The oscillator parameters resulting from the fit are listed in Table 1 and used again to calculate the RAS spectrum (red curve in Figure 3a) as a final countercheck.

On a purely $2 \mathrm{D}$ porphyrin layer, the discussed spectral analysis definitively clarifies that, on the one hand (experiment), the RAS signal line shape is due to optical transitions (interpreted in terms of LOs) placed along orthogonal directions and found at different wavelengths. On the other (ab initio calculation), two distinct optical transitions (the one at higher wavelength polarized along the fixed $\mathrm{H}-\mathrm{H}$ tautomer direction), already expected in a single $\mathrm{H}_{2}$ TPP molecule, ${ }^{25}$ also characterize the optical properties of the entire $2 \mathrm{D}$ porphyrin layer. Consequently, RAS analysis fully confirms the presence 
of frozen porphyrin tautomers at $\mathrm{RT}^{29}$ The optical properties of a given tautomer can be now thought in terms of two mutually perpendicular LOs, which resonate at distinct wavelengths because of the reduced symmetry of a frozen tautomer. Conversely, if the porphyrin tautomerism was active, the optical spectrum should be characterized, for each light polarization, by a superposition of the two LOs, due to a statistically equivalent distribution of tautomers along the two orthogonal directions. This would provide a null RAS signal and an overall isotropic optical behavior of the organic film.

The RAS spectra of three samples, not exposed to cycles of $\mathrm{HCl}$ vapors, of $0.2 \mathrm{~nm}, 1 \mathrm{~nm}$ (the same as in Figure $2 \mathrm{a}$ ), and 5 $\mathrm{nm}$ are shown in Figure 4, together with the corresponding AFM images, clearly revealing a mixed 2D-3D morphology. The RAS spectra are different for films with different thicknesses, in both line shape and intensity. Bearing in mind the above analysis, and using the RAS spectrum of the $2 \mathrm{D}$ phase as a reference signal, we address the interpretation of Figure 4 by considering that the response of the 0.2 -nm-thick film is similar but not identical to the one of the purely $2 \mathrm{D}$ phase (see Figure 4a). This suggests on the one hand the porphyrin wetting layer still gives a visible contribution to the optical properties of the film as compared to the $3 \mathrm{D}$ phase but, on the other hand, the possible presence of sample areas covered by a multilayered $2 \mathrm{D}$ phase. The latter occurrence precludes an easy interpretation of the optical spectra in terms of Lorentz oscillators and rather requires the use of a phenomenological approach. To this aim, we fit the main peak of the RAS spectra with Voigt functions, which are characteristic function profiles used in spectroscopy when many Lorentz oscillators, at slightly different frequencies, contribute to the spectral line shape. Although such an approach does not provide any description of the physical phenomena determining the optical response of the porphyrin layers, it might offer the possibility of decoupling the $2 \mathrm{D}$ and $3 \mathrm{D}$ contributions in the RAS spectra when both phases are simultaneously present. In this analysis, we reduce the fitting spectral range because the negative peak in the typical differential line shape of the RAS spectra (see Figure 4) shows a random evolution as a function of film thickness (see ref 19 and references therein). This fact suggests a different origin for this feature with respect to that obtained on the ultrathin film. On this basis, the spectrum in Figure 4a is fitted by using a Voigt curve from 410 to $440 \mathrm{~nm}$ and the best fit curve is reported as a red line superimposed to the experimental data; the corresponding fit parameters listed in Table $1\left(\mathrm{~V}_{2 \mathrm{D}}\right.$ contribution). Similarly, to account for the response of the $3 \mathrm{D}$ phase, where crystallites (structure unknown) with different size and orientation characterize the film morphology, making modeling nearly impossible, we fit the RAS spectrum of the $5 \mathrm{~nm}$ film in Figure $4 \mathrm{c}$ with a different Voigt function, here interpreted as mostly due to the $3 \mathrm{D}$ phase. The best fit curve is reported as a red line superimposed to the experimental data (Figure 4c), with the corresponding parameters listed in Table 1 ( $\mathrm{V}_{3 \mathrm{D}}$ contribution). It is interesting to note that both the 0.2 -nm-thick film ( $2 \mathrm{D}$ phase modified) and the 5-nm-thick sample (prevailing 3D phase) are fitted just by using a single Voigt curve, suggesting a strategy to analyze also films where neither of the two phases dominates. This is the case of the 1-nm-thick film, whose RAS spectrum in Figure $4 \mathrm{~b}$ indeed presents a composite line shape and a surface morphology with rather big $3 \mathrm{D}$ crystallites. The fitting of the main peak of the RAS spectrum requires two Voigt curves, one for the $2 \mathrm{D}$ phase and the other for the $3 \mathrm{D}$ phase $\left(\mathrm{V}_{2 \mathrm{D}}\right.$ and $\mathrm{V}_{3 \mathrm{D}}$, respectively). The result is shown as a red line in Figure $4 b$, in close agreement with the experimental data. The $V_{2 D}$ contribution is the same obtained for the 0.2 -nm-thick film, suggesting that the optical response of the $2 \mathrm{D}$ phase is unaffected. The spectral position of $\mathrm{V}_{3 \mathrm{D}}$ is very close to that for the 5-nm-thick film, but it shows a much lower width and intensity; this demonstrates how the $3 \mathrm{D}$ crystallites response starts to be detectable only at this thickness regime. In conclusion, the discussed analysis suggests that RAS spectra acquired on thicker films with respect to the thinnest sample can be simply interpreted in terms of a linear combination of characteristic optical responses of the $2 \mathrm{D}$ and $3 \mathrm{D}$ phases. However, while the $2 \mathrm{D}$ contribution is stable in spectral position and intensity, the $3 \mathrm{D}$ signal intensity changes as a consequence of the progressive enrichment of porphyrin 3D structures on the sample surface.

\section{CONCLUSIONS}

The RAS optical spectra of $\mathrm{H}_{2}$ TPP films grown on HOPG at $\mathrm{RT}$ are analyzed on the basis of the AFM surface images, where the coexistence of $2 \mathrm{D}$ and $3 \mathrm{D}$ phases is observed. For the purely $2 \mathrm{D}$ phase, the optical properties of the film are interpreted as a consequence of porphyrin frozen tautomers. In thicker films, where also 3D crystallites are observed by AFM, the RAS signal can be regarded as a linear superposition of the characteristic optical anisotropy spectrum of the 2D phase, always present, and a contribution arising from the $3 \mathrm{D}$ phase, with different relative weights for different film thicknesses. A close link is therefore established between the macroscopic optical anisotropy, detected by RAS, and the molecular aggregation characterizing the film morphology, a worthy step toward the fine control over the nanometric-scale sample properties needed for the development of organic nanotechnology.

\section{AUTHOR INFORMATION \\ Present Address}

European Theoretical Spectroscopy Facility (ETSF).

\section{Notes}

The authors declare no competing financial interest.

\section{ACKNOWLEDGMENTS}

The authors kindly acknowledge S. Cirilli, R. Yivlialin, A. Violante, and R. Paolesse for useful discussions and support. M.P. and C.H. acknowledge the CINECA award under the ISCRA initiative (2012-2013) for the availability of high performance computing resources and support. Part of the work was performed as a User Project (No. 478) of the European Theoretical Spectroscopy Facility (www.etsf.eu).

\section{REFERENCES}

(1) Jurow, M.; Schuckman, A. E.; Batteas, J. D.; Drain, C. M. Porphyrins as Molecular Electronic Components of Functional Devices. Coord. Chem. Rev. 2010, 254, 19-20.

(2) Auwärter, W.; Seufert, K.; Bischoff, F.; Ecija, D.; Vijayaraghavan, S.; Joshi, S.; Klappenberger, F.; Samudrala, N.; Barth, J. V. A SurfaceAnchored Molecular Four-Level Conductance Switch Based on Single Proton Transfer. Nat. Nanotechnol. 2012, 7, 41-46.

(3) Liu, Z.; Yasseri, A. A.; Lindsey, J. S.; Bocian, D. F. Molecular Memories that Survive Silicon Device Processing and Real-Word Operation. Science 2003, 302, 1543-1545.

(4) Li, C.; Ly, J.; Lei, B.; Fan, W.; Zhang, D.; Han, J.; Meyyappan, M.; Thompson, M.; Zhou, C. Data Storage Studies on Nanowire 
Transistors with Self-Assembled Porphyrin Molecules. J. Phys. Chem. B 2004, 108, 9646-9649.

(5) Haddad, R. E.; Gazeau, S.; Pécaut, J.; Marchon, J. C.; Medforth, C. J.; Shelnutt, J. A. Origin of the Red Shifts in the Optical Absorption Bands of Nonplanar Tetraalkylporphyrins. J. Am. Chem. Soc. 2003, 125, 1253-1268.

(6) Yella, A.; Lee, H.-W.; Tsao, H. N.; Yi, C.; Chandiran, A. K.; Nazeeruddin, Md. K.; Diau, E. W.-G.; Yeh, C.-Y.; Zakeeruddin, S. M.; Grätzel, M. Porphyrin-Sensitized Solar Cells with Cobalt (II/III)based Redox Electrolyte Exceed 12\% Efficiency. Science 2011, 334, 629-634.

(7) Santonico, M.; Lucantoni, G.; Pennazza, G.; Capuano, R.; Galluccio, G.; Roscioni, C.; La Delfa, G.; Consoli, D.; Martinelli, E.; Paolesse, R; et al. In Situ Detection of Lung Cancer Volatile Fingerprints Using Bronchoscopic Air-Sampling. Lung Cancer 2012, $77,46-50$.

(8) Bussetti, G.; Violante, A.; Yivlialin, R.; Cirilli, S.; Bonanni, B.; Chiaradia, P.; Goletti, C. Site-Sensitive Gas Sensing and Analyte Discrimination in Langmuir-Blodgett Porphyrin Films. J. Phys. Chem. C 2011, 115, 8189-8194.

(9) Hulsken, B.; Van Hameren, R.; Gerritsen, J. W.; Khoury, T.; Thordarson, P.; Crossley, M. J.; Rowan, A. E.; Nolte, R. J. M.; Elemans, J. A. A. W.; Speller, S. Real-Time Single-Molecule Imaging of Oxidation Catalysis at a Liquid-Solid Interface. Nat. Nanotechnol. 2007, 2, 285-289.

(10) Otsuki, J. STM Studies on Porphyrins. Coord. Chem. Rev. 2010, 254, 2311-2341.

(11) Iancu, V.; Deshpande, A.; Hla, S. W. Manipulating Kondo Temperature via Single Molecule Switching. Nano Lett. 2006, 6, 820823.

(12) Wende, H.; Bernien, M.; Luo, J.; Sorg, C.; Ponpadian, N.; Kurde, J.; Miguel, J.; Piantek, M.; Xu, X.; Eckhold, Ph.; et al. SubstrateInduced Magnetic Ordering and Switching of Iron Porphyrin Molecules. Nat. Mater. 2007, 6, 516-520.

(13) Drain, C. M.; Mauzerall, D. An Example of a Working Molecular Charge Sensitive Ion Conductor. J. Electroanal. Chem. Interfacial Electrochem. 1990, 299, 263-268.

(14) Liu, C. Y.; Pan, H. I.; Fox, M. A.; Bard, A. J. High-Density Nanosecond Charge Trapping in Thin Films of the Photoconductor ZnODEP. Science 1993, 261, 897-899.

(15) Hassani, S. S.; Kim, Y. G.; Borguet, E. Self-Assembly of Insoluble Porphyrins on $\mathrm{Au}(111)$ under Aqueous Electrochemical Control. Langmuir 2011, 27, 14828-14833.

(16) Heim, D.; Écija, D.; Seufert, K.; Auwärter, W.; Aurisicchio, C.; Fabbro, C.; Bonifazi, D.; Barth, J. V. Self-Assembly of Flexible OneDimensional Coordination Polymers on Metal Surfaces. J. Am. Chem. Soc. 2010, 132, 6783-6790.

(17) Song, C. H.; Back, S. Y.; Yu, S. I.; Lee, H. J.; Kim, B. S.; Yang, N. Y.; Jeong, S. H.; Ahn, H. Direct Patterning of Porphyrin Dot Arrays and Lines Using Electrohydrodynamic Jet Printing. J. Nanosci. Nanotechnol. 2012, 12, 475-480.

(18) Forker, R; Gruenewald, M.; Fritz, T. Optical Differential Reflectance Spectroscopy on Thin Molecular Films. Annu. Rep. Prog. Chem., Sect. C: Phys. Chem. 2012, 108, 34-68. Papageorgiou, N.; Salomon, E.; Angot, T.; Layet, J.-M.; Giovanelli, L.; Le Lay, G. Physics of Ultra-Thin Phthalocyanine Films on Semiconductors. Prog. Surf. Sci. 2004, 77, 139-170.

(19) Goletti, C.; Bussetti, G.; Chiaradia, P.; Sassella, A.; Borghesi, A. The Application of Reflectance Anisotropy Spectroscopy to Organics Deposition. Org. Electron. 2004, 5, 73-81.

(20) Qiu, X.; Wang, C.; Zeng, Q.; Xu, B.; Yin, S.; Wang, H.; Xu, S.; Bai, C. Alkane-Assisted Adsorption and Assembly of Phthalocyanines and Porphyrins. J. Am. Chem. Soc. 2000, 122, 5550-5556.

(21) Müllegger, S.; Rashidi, M.; Lengauer, T.; Rauls, E.; Schmidt, W. G.; Knör, G.; Schöfberger, W.; Koch, R. Asymmetric Saddling of Single Porphyrin Molecules on Au(111). Phys. Rev. B 2011, 83, 165416-(14).

(22) Auwärter, W.; Weber-Bargioni, A.; Brink, S.; Riemann, A.; Schiffrin, A.; Ruben, M.; Barth, J. V. Controlled Metalation of Self-
Assembled Porphyrin Nanoarrays in Two Dimensions. ChemPhysChem 2007, 8, 250-254.

(23) Buchner, F.; Schwald, V.; Comanici, K.; Steinrück, H.-P.; Marbach, H. Microscopic Evidence of the Metalation of a Free-Base Porphyrin Monolayer with Iron. ChemPhysChem 2007, 8, 241-243.

(24) Hennig, J.; Limbach, H.-H. Kinetic Study of Hydrogen Tunneling in Meso-Tetraphenylporphine by Nuclear Magnetic Resonance Lineshape Analysis and Selective T1 $\rho$-Relaxation Time Measurements. J. Chem. Soc., Faraday Trans. 1979, 75, 752-766.

(25) Palummo, M.; Hogan, C.; Sottile, F.; Bagalá, P.; Rubio, A. Ab Initio Electronic and Optical Spectra of Free-Base Porphyrins: the Role of Electronic Correlation. J. Chem. Phys. 2009, 131, 084102(17).

(26) Wehrle, B.; Limbach, H.-H.; Köcher, M.; Ermer, O.; Vogel, E. N-CPMAS-NMR Study of the Problem of NH Tautomerism in Crystalline Porphine and Porphycene. Angew. Chem. 1987, 99, 914916.

(27) Scarselli, M.; Ercolani, G.; Castrucci, P.; Monti, D.; Bussetti, G.; Russo, M.; Goletti, C.; Chiaradia, P.; Paolesse, R.; De Crescenzi, M. A Combined Scanning Tunneling Microscopy and Reflectance Anisotropy Spectroscopy Investigation of Tetraphenylporphyrin Deposited on Graphite. Surf. Sci. 2007, 601, 2607-2610.

(28) Campione, M.; Fumagalli, E.; Raimondo, L.; Monguzzi, A.; Meinardi, F.; Sassella, A. Control of $\pi$ - $\pi$ Interactions in Epitaxial Films of Platinum (II) Octaethylporphyrin. Chem. Mater. 2011, 23, 832840.

(29) Bussetti, G.; Campione, M.; Riva, M.; Picone, A.; Raimondo, L.; Ferraro, L.; Hogan, C.; Palummo, M.; Brambilla, A.; Finazzi, M.; et al. Stable Alignment of Tautomers at Room Temperature in Porphyrin 2D Films. Adv. Funct. Mater. 2014, 24, 958-963.

(30) Sassela, A.; Ferraro, L.; Bussetti, G. Metodo e apparato di spettroscopia in riflettanza anisotropa. Patent pending. MI2013A002184.

(31) Weightman, P.; Martin, D. S.; Cole, R. J.; Farrell, T. Reflection Anisotropy Spectroscopy. Rep. Prog. Phys. 2005, 68, 1251.

(32) Marini, A.; Hogan, C.; Grüning, G.; Varsano, D. An Ab Initio Tool for Excited State Calculations. Comput. Phys. Commun. 2009, $180,1392-1403$.

(33) Hogan, C.; Palummo, M.; Gierschner, J.; Rubio, A. Correlation Effects in the Optical Spectra of Porphyrin Oligomer Chains: Exciton Confinement and Length Dependence. J. Chem. Phys. 2013, 138, 024312.

(34) Onida, G.; Reining, L.; Rubio, A. Electronic Excitations: Density-Functional Versus Many-Body Green's-Function Approaches. Rev. Mod. Phys. 2002, 74, 601-659.

(35) Bussetti, G.; Campione, M.; Raimondo, L.; Yivlialin, R.; Finazzi, M.; Ciccacci, F.; Sassella, A.; Duò, L. Unconventional Post-Deposition Chemical Treatment on Ultra-Thin $\mathrm{H}_{2}$ TPP Film Grown on Graphite. Cryst. Res. Technol. 2014, DOI: 10.1002/crat.201300406.

(36) De Luca, G.; Romeo, A.; Monsù Scolaro, R.; Ricciardi, G.; Rosa, A. Evidence of Tetraphenylporphyrin Monoacids. Inorg. Chem. 2007, 46, 5979-5988.

(37) McIntyre, J. D. E.; Aspnes, D. E. Differential Reflection Spectroscopy of Very Thin Surface Films. Surf. Sci. 1971, 24, 417-434.

(38) Palik, E. D. Handbook of Optical Constants of Solids; Academic Press: New York, 1998.

(39) Wooten, F. Optical Properties of Solids; Academic Press: New York, 1972. 